\title{
Affective biasing of choices in gambling task decision making
}

\author{
JOHN M. HINSON, PAUL WHITNEY, HEATHER HOLBEN, and AARON K. WIRICK \\ Washington State University, Pullman, Washington
}

\begin{abstract}
The proponents of the somatic marker hypothesis presume that rational decision making is guided by emotional reactions that are developed from prior experience. Supporting evidence for the hypothesis comes almost exclusively from the short-term affective reactions that are learned during the course of a hypothetical decision-making task - the gambling task (GT). We examined GT performance and affective reactions to choices when those choices were biased by words that had preexisting affective value. In one experiment, affectively valued words directly signaled good and bad choices. A congruent relation between affective value of word and choice outcome improved GT performance, whereas an incongruent relation greatly interfered with performance. In another experiment, affectively valued words were maintained as a working memory (WM) load between GT choices. A WM load with affectively positive words somewhat improved GT performance, whereas affectively negative words interfered with performance. Somatic markers-indicated by differential anticipatory skin conductance response (SCR) amplitude for good and bad choices-appeared at a point in the GT session when choice performance was superior. However, differential SCR developed during the session after good choice performance was already established. These results indicate that preexisting affective biases can influence GT decision making. In addition, the somatic markers that are regular accompaniments of GT decision making appeared to be temporally lagging indicators of choice performance.
\end{abstract}

Since the beginnings of scientific psychology, there has been an ongoing argument about the causal relations among emotion, cognition, and action (see, e.g., Dolan, 2002). This topic has been revitalized over the past decade by the introduction of the somatic marker hypothesis proposed by A. R. Damasio (e.g., 1994). The somatic marker hypothesis was put forth to try to resolve the century-old debate over the causal role of emotion by reasserting the preeminence of emotion in decision making. The proponents of the hypothesis posit that conscious decision making is guided by the affective reactions that typically develop automatically and unconsciously from the consequences of prior actions. Accordingly, affective biases precede and facilitate the conscious decision-making processes that rationally evaluate the outcomes of actions. More simply put, under normal circumstances, people will feel that a choice of action is good or bad before they actually know why that choice is good or bad. Moreover, Damasio and colleagues argue that people who make poor decisions often do so because they lack the emotional reactions that signal a bad outcome from a pending choice (Bechara, Dolan, \& Hindes, 2002; A. R. Damasio, 1994).

The most compelling evidence for the somatic marker hypothesis is the seemingly unique pattern of decision making and affective reactions found in patients with damage to the ventromedial prefrontal cortex (VMPFC). In their

Address correspondence to J. M. Hinson, Department of Psychology, Washington State University, Pullman, WA 99164-4820 (e-mail: hinson@ mail.wsu.edu). studies of VMPFC patients, Damasio, Bechara, and colleagues have used skin conductance response (SCR) as a measure of affective response during a simulated gambling task (GT) (see, e.g., Bechara, H. Damasio, \& A. R. Damasio, 2000; Bechara, Tranel, H. Damasio, \& A. R. Damasio, 1996; A. R. Damasio, Tranel, \& H. Damasio, 1991). The GT is a simulated card game in which a person begins with a hypothetical stake of money and makes choices among four decks, two of which are relatively good and two of which are relatively bad. Each choice can produce gains and losses in varying amounts. In the good decks, gains outweigh losses in the long run, whereas in the bad decks, losses outweigh gains in the long run (Bechara, A. R. Damasio, H. Damasio, \& Anderson, 1994).

VMPFC patients perform poorly on the GT by selecting the bad decks, which produce large short-term gains but even larger long-term losses. Along with these suboptimal GT choices, VMPFC patients do not show anticipatory SCR to either good or bad choices. Although they show affective reactions to both good and bad outcomes, these patients do not anticipate the deleterious consequences of actions (Bechara, H. Damasio, Tranel, \& A. R. Damasio, 1997). One interpretation of these results is that VMPFC patients do not lack emotion, but rather lack the somatic markers that ordinarily guide decision making. A more general conclusion that may be drawn from these studies is that the VMPFC is an important component of the decision-making network that directs the activation of emotional biases, which are based on individual experience in comparable decision-making situations in the past. On the basis of evidence from decision making in these 
special cases of orbitofrontal cortex injury, and on the basis of the interpretation of this evidence as it regards the specialization of the VMPFC pathway, the proponents of the somatic marker hypothesis make broad claims about how suboptimal decision making arises (Bechara, 2004).

Several critics of the somatic marker hypothesis have questioned the role of covert affective biasing in decision making (Maia \& McClelland, 2004; Rolls, 2004). In early studies of the somatic marker hypothesis, emphasis was placed on the fact that anticipatory SCR to good and bad choices in normal control participants developed before these people could consciously report or conceptualize the GT contingencies (Bechara et al., 1997). The assumption that the GT is not cognitively penetrable makes it more plausible that affective processes, such as somatic markers, would play a causal role in decision making (see, e.g., Dunn, Dalgleish, \& Lawrence, 2006). However, since the early reports, a more detailed study has examined what people are able to report about their conscious knowledge of the GT and how this knowledge is related to performance on the GT (Maia \& McClelland, 2004). Non-braininjured participants who perform well on the GT appear to have explicit knowledge of GT contingencies. Furthermore, this knowledge accrues much earlier in the GT session than previously suspected, making it possible that somatic markers result from explicit knowledge of choice outcomes. According to critics, these findings undermine the claim that somatic markers precede and direct GT decision making in populations without VMPFC damage (see Dunn et al., 2006).

In response, advocates of the somatic marker hypothesis have noted that people may accurately report knowledge of choice outcomes and still perform poorly on the GT (Bechara, H. Damasio, Tranel, \& A. R. Damasio, 2005). Therefore, explicit knowledge of choice contingencies does not preclude a role for somatic markers. Moreover, there is still a debate about what participants actually know, and whether it qualifies as a hunch or as full conceptual knowledge (Bechara et al., 2005). The most recent interpretation of the somatic marker hypothesis includes the suggestion that, even when there is explicit knowledge of contingencies, somatic markers may still develop and influence subsequent decision making. From this point of view, what is important is that affective reactions are being triggered during the deliberation of choice outcomes and that a person may not be consciously aware of these affective reactions. Once triggered, an affective reaction can modulate choice without the person's awareness, and this may or may not occur in concert with other explicit knowledge of choice outcomes (Bechara \& A. R. Damasio, 2005).

The suggestion that an explicit understanding of the contingencies involved in the GT may be needed for good performance is consistent with neurological and behavioral evidence that participants must actively maintain information in working memory (WM) to perform well on the task. Patients with damage to the dorsolateral prefrontal cortex (DLPFC), which is involved in a wide range of WM tasks, show poor GT performance (Manes et al., 2002). Moreover, people with injury to both the VMPFC and the DLPFC perform even worse (Manes et al., 2002). Furthermore, work with neurologically intact people demonstrates that loading WM not only disrupts GT performance but also interferes with the development of anticipatory affective reactions (Hinson, Jameson, \& Whitney, 2002). The data on WM and the GT do not refute the claim by Damasio and colleagues that decision making is biased by the somatic markers that are produced by a dissociable neural circuit (Bechara, H. Damasio, Tranel, \& A. R. Damasio, 1998). But there must be, at least, a strong interdependence between the VMPFC affective processes and the WM processes of the DLPFC in this decision-making setting. Indeed, Damasio (1994; see also Bechara \& A. R. Damasio, 2005) has suggested that somatic markers may serve as signals that exert control over which information gets priority for processing in WM.

Regardless of how one views the relation between explicit knowledge of task contingencies and somatic markers, the somatic marker hypothesis provides an account of affective processes that could occur when there are deviations between knowledge and action, or, conversely, when knowledge and action are consistent. Regardless of how quickly explicit knowledge arises, according to the advocates of the somatic marker hypothesis, the presumption is that affective biases influence decision making, covertly or overtly. Prior studies have examined how affective markers arise during the brief course of learning choice outcomes in the GT. However, it is not clear whether these affective reactions influence decision making or whether they are a somatic response to making a decision and anticipating a consequence (see Dunn et al., 2006). The present experiments examined possible changes in the quality of GT decision making when emotional reactions were directly manipulated, independently of the outcome of each GT choice. We hypothesized that manipulation of affect orthogonal to gains and losses in the GT would provide more direct information about the causal role of affective processes in this decision-making setting.

\section{EXPERIMENT 1}

To investigate the role of affective biases in decision making, we used a modified version of the GT that allowed us to manipulate affective reactions to the decks independently of the gains and losses produced by the choices. As in previous versions of the GT, participants learned to choose good and bad outcomes from among various options. However, the version of the GT that we used did not script the outcomes so that the bad decks started with a series of high rewards, as the original GT did. Thus, the results we obtained from the decision-making procedure that we used are not the product of a particular sequence of outcomes. Nevertheless, we have previously shown that our version of the GT produces both behavioral data and somatic markers that are comparable to data from the original GT (Hinson et al., 2002; Jameson, Hinson, \& Whitney, 2004).

In Experiment 1, words with preestablished emotional value were presented along with the GT choices. We 
tested whether the emotional value of the words would contribute to, or interfere with, the underlying emotional reactions that normally develop during the GT.

\section{Method}

\section{Participants}

The participants were 70 undergraduate students at Washington State University who received extra course credit. They were from 18 to 25 years old and were approximately $60 \%$ female.

\section{Procedure}

The participants were given a brief oral description of the task, paraphrased as follows:

In this experiment, you will be asked to make hypothetical gambles similar to those made in a card game. You will start with a fixed sum of money, and the computer will prompt you to make repeated choices. For each choice, you will sometimes lose money and sometimes win money. Your task is to discover the best choices to make so that at the end of the session you will have the highest amount of money possible. Each choice will be identified with a specific number and each will be labeled with a different word. When you make your choice on each trial, please press on the appropriate keypad number and say the word that identifies the choice out loud.

The participants were seated in front of individual computer terminals and told to begin when ready. A program written in Microsoft Basic provided written instructions and practice trials to illustrate the procedure and collected all the data from the experiment. The participants were given four practice trials to familiarize themselves with the procedure.

Each participant was given 100 GT trials. On each trial, the computer gave a choice among three options. These options were displayed as rectangles, each roughly the area of a playing card, colored violet, with the number 1, 2, or 3 in the middle. Outcomes for choices were based on a fixed set of gains and losses. Selection among these outcomes was based on values from a random number generator that provided random numbers from a uniform probability distribution. One choice, the good deck, produced small gains with even smaller losses over repeated choices $(0,25,50,-10$, or -25 on any given trial). Another choice, the neutral deck, resulted in neither gains nor losses over repeated choices $(0,75,-50,-75$, or -100 on any given trial). The third choice, the bad deck, produced large gains with even larger losses over repeated choices $(100,-50$, $-150,-200$, or -250 on any given trial). Table 1 provides a summary of the choice outcomes, arranged for the three decks.

As in our previous work, the computer-simulated decks in Experiment 1 were presented in a consistent screen location with a corresponding keypad response - that is, left (Response 1), center (Response 2), or right (Response 3). The position of good, bad, and neutral decks was randomized across participants, who indicated their choice on each trial by pressing the numeric key associated with the deck of their choice on that trial.

The decks were labeled with numbers as well as words that differed in emotional valence - positive, negative, or neutral - according to the ratings established in the Affective Norms for English Words system (Bradley \& Lang, 1999). Positive and negative words fell between 1 and $2 S D$ s away from affectively neutral, whereas neutral words fell within $1 S D$ away from affectively neutral. Words in the three emotional valence categories were equated for general arousal

Table 1

Scheduled Payoff Parameters for Choices in Experiment 1

\begin{tabular}{lrrrrc}
\hline Choice Type & Min & Max & \multicolumn{1}{c}{$M$} & $S D$ & $p$ (loss) \\
\hline Good & -25 & 50 & 25 & 28 & .2 \\
Neutral & -100 & 75 & 0 & 67 & .5 \\
Bad & -250 & 100 & -25 & 135 & .5 \\
\hline
\end{tabular}

and frequency of occurrence in the English language. A unique set of 100 words was selected in each category to avoid any spurious contingency arising from the recognition of repeated words during the session. In addition to signaling their choice with a numeric key, the participants were to say the word from the chosen deck aloud to ensure that they were attending to the word as well as to the screen location and the keypad response number.

The participants were randomly assigned to one of two conditionscongruent or incongruent. For the congruent condition, affectively positive words appeared on the good deck, affectively negative words appeared on the bad deck, and affectively neutral words appeared on the neutral deck. In the incongruent condition, affectively positive words appeared on the bad deck, affectively negative words appeared on the good deck, and affectively neutral words appeared on the neutral deck.

SCR measurement. SCR was recorded by means of a Contact Precision Instruments SC5 SA skin conductance monitor. We were primarily interested in anticipatory SCR - the affective response that occurs before a gambling choice. To analyze affective responsiveness, we measured changes in SCR amplitude (see, e.g., Dawson, Schell, \& Filion, 2000; Hinson et al., 2002). At the beginning of each trial, the skin conductance level (SCL) was sampled to establish the baseline level for that trial. The baseline SCL value was the mean for SCL samples taken during the beginning $0.5 \mathrm{sec}$ of the trial. SCL was then continuously sampled until a gambling choice was made to determine peak SCL. The SCR amplitude for the trial was calculated as the difference between peak SCL and baseline SCL on that trial. Whenever there was a difference between peak SCL and baseline SCL of less than $0.01 \mu \mathrm{S}$, no response on that trial was considered to have occurred. Further details of the recording procedure may be found in Hinson et al. ${ }^{1}$

This SCR measure was designed to show the anticipatory affective response before the outcome of the choice on that trial is known (see, e.g., Bechara et al., 1997). Here we used SCR amplitude as an index of emotional arousal during the period in which the participant was deliberating choices, just as in earlier studies. Note, however, that there was no way to know precisely what was being deliberated. In the earlier GT studies, four choice options were concurrently available. Because the SCR that occurs during deliberation is associated with a choice only after that choice is made, we cannot know how many different choices were actually being deliberated, only that a particular choice was ultimately made. In Experiment 1, we measured the prevailing affective reaction during the deliberation period as a combined function of anticipation of choice outcome and the preexisting affective reaction to the word. In the GT, there is no obvious way to disentangle the separate contributions of choice outcome and affective value of the word.

The timing of the SCR amplitude depended on the latency of each participant's choices during the GT trial. The sequence of trial events was as follows: 1.5-sec delay-2-sec wait-GT choice (keypad and spoken word)-1.5-sec tally display.

\section{Results and Discussion}

Figure 1 summarizes GT performance. The figure gives the proportion of good, bad, and neutral choices in each of four blocks of 25 trials for the two signaling conditions, congruent and incongruent. Participants very rapidly learned to make good choices in the congruent condition, with slight improvement throughout the remaining blocks of trials. Participants in the incongruent condition showed very poor initial performance. Bad choices predominated initially and slowly declined across blocks of trials. Performance in the final block of trials in the incongruent condition remained strikingly suboptimal, with bad choices continuing to outnumber good choices. Statistical 


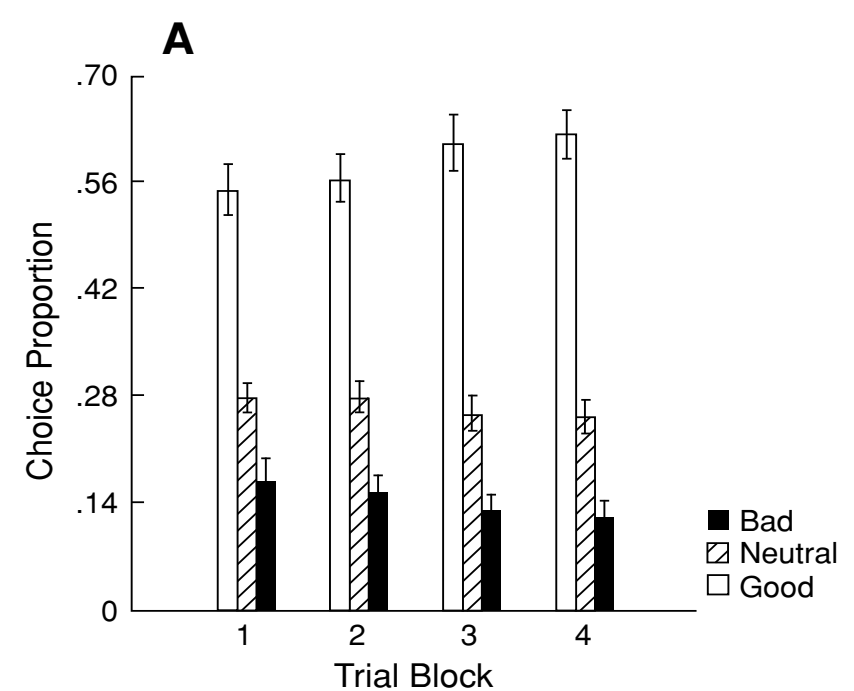

B

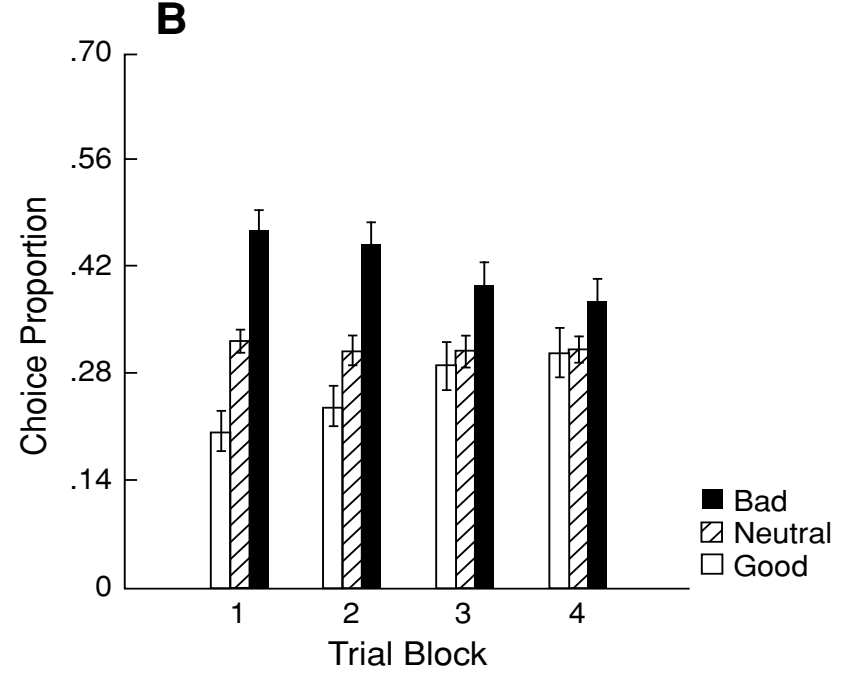

Figure 1. Mean proportion and $S E M$ for the three GT choice types across four blocks of 25 trials in Experiment 1. (A) Congruent condition. (B) Incongruent condition.

reliability of the GT choice results, with alpha level of .05 for this and all subsequent analyses, was confirmed by means of a 2 (congruency condition) $\times 2$ (good or bad choice type) $\times 4$ (trial block) repeated measures ANOVA. The ANOVA indicated main effects of congruency condition $[F(1,68)=5.359]$ and choice type $[F(1,68)=17.72]$. There were also significant interactions between choice type and congruency condition $[F(1,68)=103.54]$ and between trial block and choice type $[F(3,204)=8.765]$. Considered alone, good choices increased across blocks of trials in both the congruent condition $[F(3,102)=3.054]$ and the incongruent condition $[F(3,102)=5.465]$. Paired $t$ tests showed that good choices exceeded bad choices in all trial blocks for the congruent condition, whereas good choices never predominated in any trial block for the incongruent condition. Thus, the emotional valence of the words on the decks had a profound effect on GT performance. The congruent condition facilitated GT per- formance, whereas the incongruent condition interfered with GT performance. ${ }^{2}$

Figure 2 shows SCR amplitude prior to the selection of each GT choice. Note that we are focusing on differential SCR amplitude rather than absolute SCR. Absolute SCR may be subject to many extraneous influences that have nothing to do with discrimination of choice outcomes in the GT (see, e.g., Dawson et al., 2000). Also, the absolute magnitude of SCR for good and bad choices depends on the features of the payoff schedules, not the category of good or bad choice (Hinson et al., 2002; Tomb, Hauser, Deldin, \& Caramazza, 2002). For these and other reasons, we used differential SCR amplitude as the most appropriate measure of affective state in GT studies. Unless a participant is having a differential response to good and bad choices, affective state cannot be guiding decision making (see Maia \& McClelland, 2004).

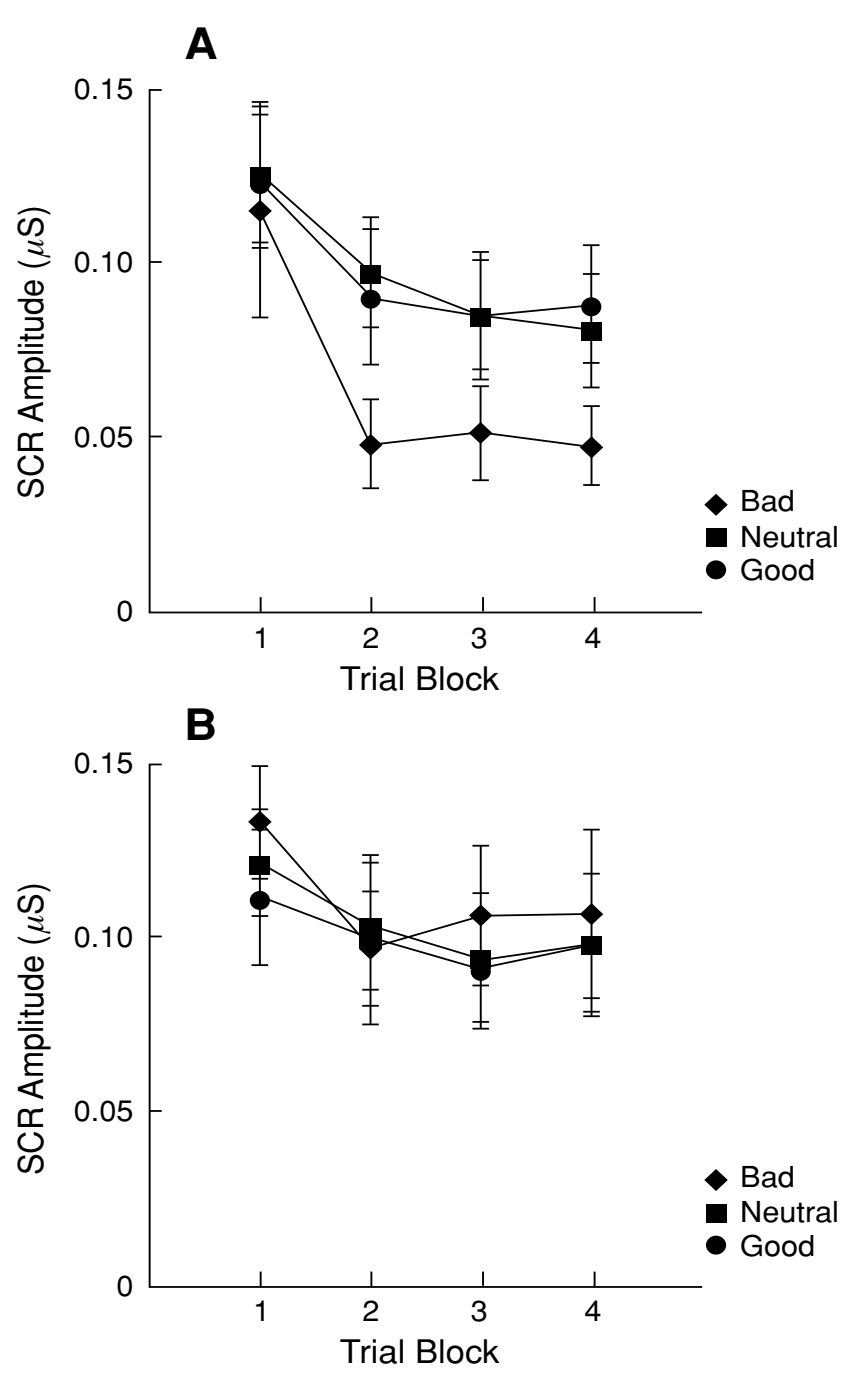

Figure 2. Mean anticipatory SCR amplitude and SEM for the three GT choice types across four blocks of 25 trials in Experiment 1. (A) Congruent condition. (B) Incongruent condition. 
For the incongruent condition, overall SCR amplitude was high, but there was no differential response to good and bad choices. As in earlier work, the absence of a differential affective response was associated with poor GT performance. By comparison, in the congruent condition, differential anticipatory SCR amplitude developed across blocks of trials, with SCR to good choices being larger than SCR to bad choices. The reliability of SCR results was confirmed by means of a 2 (congruency condition) $\times$ 3 (good, neutral, or bad choice type) $\times 4$ (trial block) repeated measures ANOVA. The ANOVA indicated a main effect of trial block $[F(3,204)=8.316]$. There also was a significant interaction between trial type and congruency condition $[F(2,136)=9.394]$. Paired $t$ tests showed that SCR amplitude for good choices exceeded that for bad choices in Blocks 2, 3, and 4 for the congruent condition. SCR amplitudes for good and bad choices never differed in the incongruent condition.

This pattern of SCR results is consistent with prior work indicating that good GT performance is associated with differential affective response to choices (e.g., Bechara et al., 1997; Hinson et al., 2002). However, if somatic markers were guiding decision making when participants are selecting the good option, we would expect that the somatic marker would arise prior to good performance, or at least be coincident with it. Instead, GT performance in the congruent condition was already near asymptote in the first block of 25 trials, but there was no evidence of differential affective response at that time. Differential anticipatory SCR in the congruent condition developed by Block 2 and remained throughout the session.

Thus, the somatic marker measured by SCR amplitude appeared after GT performance was already well established. The somatic marker ultimately reflected the level of GT performance, but we found no evidence that it contributed to performance. ${ }^{3}$

\section{EXPERIMENT 2}

In the congruent condition in Experiment 1, the participants appeared to be actively selecting positive words, rather than just avoiding negative words. If they were simply avoiding the negative words, they should have selected both positive and neutral words at high levels. Instead, they preferentially chose positive words from the outset. To determine whether GT performance in the congruent condition was truly facilitated by affectively positive words, we conducted a control experiment in which affectively neutral words were used for all GT choices. The orthographic features of the words were used to signal good and bad choices.

\section{Method}

\section{Participants}

The participants were 40 undergraduate students at Washington State University who received extra course credit. They were from 18 to 25 years old and were approximately $55 \%$ female.

\section{Procedure}

The participants were given instructions identical to those in Experiment 1 . The procedural features of Experiment 2 were the same as in Experiment 1, with the following exceptions: Good, bad, and neutral decks were labeled with words that differed orthographically rather than by emotional valence. The participants were randomly assigned to one of two groups: In one group, uppercase signaled good choices and lowercase signaled bad choices; in the other group, uppercase signaled bad choices and lowercase signaled good choices. Neutral choices were signaled by words that had their first letter in uppercase and the rest of their letters in lowercase. All words presented were within $1 S D$ of affective neutrality.

\section{Results and Discussion}

Because no differences were associated with the usage of upper- or lowercase words to signal good or bad choices, the data are collapsed and presented as a single control condition. Figure 3 summarizes GT performance. In the first trial block, choice proportions for the three options are roughly equivalent. During the remaining trial blocks, good choices slowly increase and diverge from bad choices. If one compares the results to those in Figure 1, GT performance in the congruent condition is superior to control, whereas GT performance in the incongruent condition is inferior to control. Statistical reliability of the GT choice results was confirmed by means of a 2 (good or bad choice type) $\times 4$ (trial block) repeated measures ANOVA. The ANOVA indicated a main effect of choice type $[F(1,39)=11.12]$. There was also a significant interaction between trial block and choice type $[F(3,117)=3.949]$. Good choices increased across trial blocks $[F(3,117)=3.711]$. Paired $t$ tests indicated that good choices did not reliably exceed bad choices until Block 3.

Figure 4 displays anticipatory SCR for the control condition. There is no evidence of differential SCR during the first two trial blocks. Differential SCR develops later and is maintained during the remaining blocks of trials. Reliability of the SCR results was confirmed by means of a 3 (good, neutral, or bad choice type) $\times 4$ (trial block) repeated measures ANOVA. The ANOVA indicated main

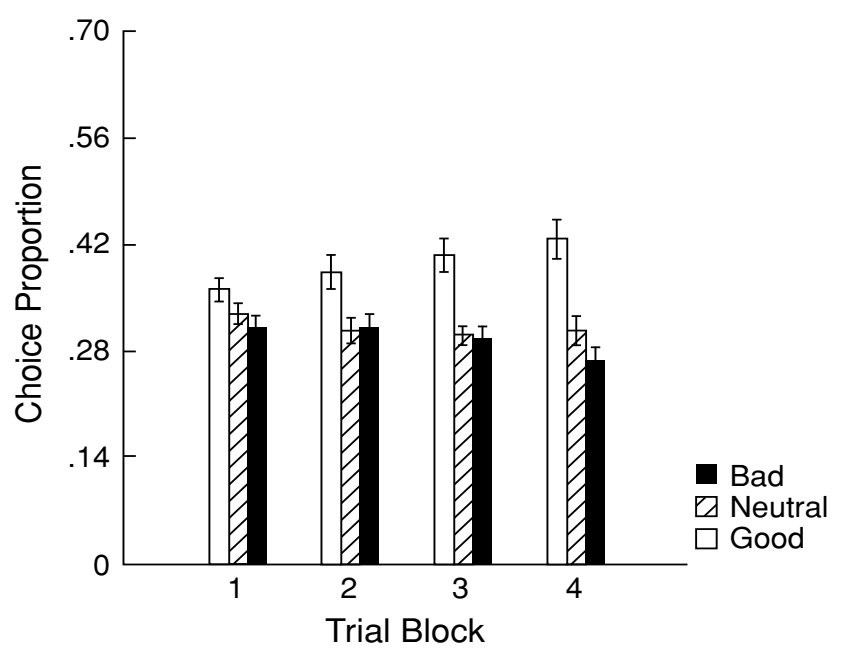

Figure 3. Mean proportion and $S E M$ for the three GT choice types across four blocks of 25 trials in Experiment 2. 


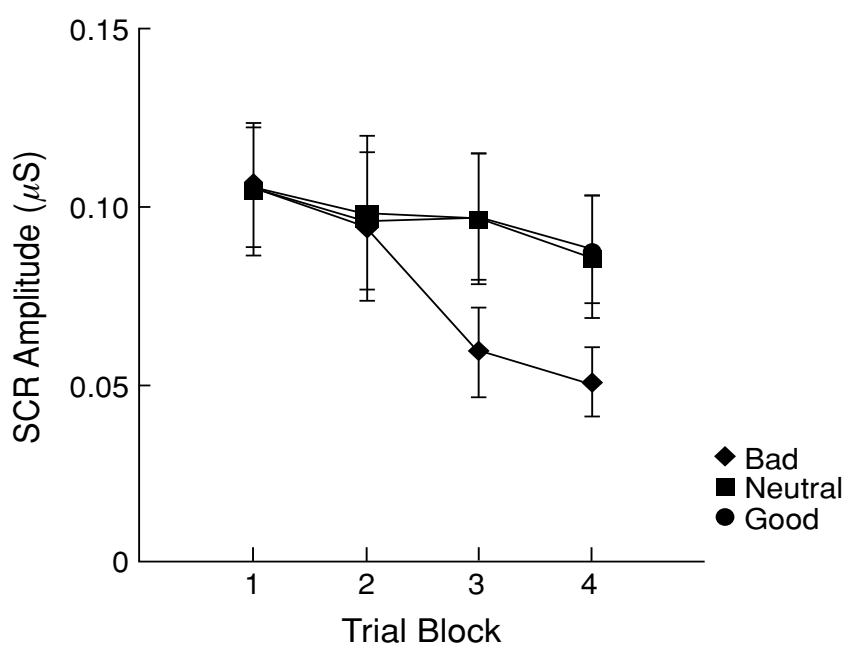

Figure 4. Mean anticipatory SCR amplitude and SEM for the three GT choice types in Experiment 2.

effects of choice type $[F(2,78)=4.507]$ and trial block $[F(3,117)=2.723]$. There was also a significant interaction between trial block and choice type $[F(6,234)=$ 2.606]. Paired $t$ tests indicated that SCR amplitude for good choices reliably exceeded that for bad choices in Blocks 3 and 4.

The results of Experiments 1 and 2 confirm the often reported finding that poor decision making on the GT is associated with the absence of an anticipatory affective reaction to choices. Poor performance throughout the incongruent condition and inferior performance during the initial blocks of the control condition were associated with the absence of differential SCR amplitude for good and bad choices.

Nevertheless, the results show no reason to conclude that the somatic marker is responsible for biasing choices toward the good option. For example, the pattern of development of differential SCR was similar for the control and congruent conditions, yet there were important differences in GT performance. If we had examined only the control condition, we would have observed a consistent relation in which good GT performance would be predicted by differential anticipatory SCR. On this basis, we might have reasonably concluded that the somatic marker was responsible for good GT performance. But a comparison of the SCR pattern in the control condition with the SCR pattern in the congruent condition suggested that differential anticipatory SCR was a reflection of good GT performance, not the cause of it. Consider the most striking comparison, in which choice performance in Block 1 of the congruent condition clearly exceeded asymptotic performance in Block 4 of the control condition. Differential SCR amplitude for good and bad choices appeared by Block 4 in the control condition. But differential SCR was clearly absent in Block 1 of the congruent condition, despite the superior GT performance. Emotional congruence of the words and the outcomes of choosing a deck resulted in better decision-making performance in the congruent condition, though not by augmenting the somatic marker. The somatic marker appeared only after good performance was well established.

The emotional incongruence of the words and the outcomes of choosing an option had even more profound effects on performance than did emotional congruence. In the incongruent condition, participants had an extremely difficult time making good choices. The words selected as positive and negative were not extreme, nor were they likely to evoke the profound emotional reactions produced with pictorial displays often used to manipulate emotion (see, e.g., Bechara et al., 2000). On the face of it, one might have expected some initial disruption, because positive words were likely to be associated with good outcomes and negative words with bad outcomes. However, after this initial disruption, it should have been an easy matter to change the response mapping so that negative words would mean a good choice and positive words would mean a bad choice. Our participants were not able to make this simple translation. During the debriefing after the session, we learned that many participants were extremely puzzled by their poor performance in the incongruent condition; they did not understand the disruptive effect of the negative words.

These results are reminiscent of an extensive body of literature on interpreting and predicting people's behavior. It is not uncommon for people to base their judgments on pseudocontingencies between evaluative dimensions (Fiedler \& Freytag, 2004). For example, in a laboratory study that simulated forming impressions of students in a classroom, the apparent levels of ability and motivation of groups of students were manipulated independently. Even when evidence showed that students being high or low in one dimension was unrelated to their level in the other dimension, participants' impressions of students were influenced as though the dimensions were related. As was the case with the good decks that had negative words in the present study, classes of students that seemed low in overall ability were rated as low in motivation, even in the face of contrary objective evidence (Fiedler, Walther, Freytag, \& Plessner, 2002). In the GT, whether a choice is labeled in negative or positive terms may bias the processing of actual contingencies, in such a way that in guiding future choices, congruent information receives more attention and more weight than incongruent information.

To summarize the results of Experiments 1 and 2, we found that, consistent with the general thrust of both the somatic marker hypothesis and other recent theories of the relationship between emotion and decision making (see, e.g., Loewenstein \& Lerner, 2003), the affective valence of words that labeled the decks had profound effects on choice behavior. However, the specific claim that the decisions were biased by somatic markers did not find support in our data, given that the somatic markers were a lagging indicator of performance. Two caveats should be noted. First, the inherent variability of SCRs may make it difficult to resolve the exact time course of the formation of a somatic marker. Second, Bechara et al. (2000) have drawn a distinction between somatic markers that involve "the body loop," which lead to peripheral effects detect- 
able with SCR, and somatic markers involving the "as if body loop," in which the processes are entirely central. ${ }^{4}$ We will address these issues further in the General Discussion section.

\section{EXPERIMENT 3}

In Experiment 1, participants had the opportunity, either overtly or covertly, to learn an association between emotion-producing words and choice outcomes. Of course, emotional reactions may occur in the decisionmaking setting for reasons that are extraneous to task requirements. These extraneous emotions and feelings would not be associated with differential gains and losses from choices that were based on prior experience in the GT. Presumably, with no predictive relation to specific choice outcomes, these emotions and feelings could not serve as somatic markers. If positive and negative emotions occur in the decision-making task, and these emotions are not signals of good and bad choices, it is not clear what impact they would have. One possibility consistent with the somatic marker hypothesis is that the presence of any significant positive or negative emotion orthogonal to decision-making task outcomes would make it harder for a person to discriminate positive and negative somatic markers. In other words, the extraneous emotion would make for a noisier internal environment, and decision making would be impaired. Another possibility is that positive emotions may enhance performance and negative emotions might depress performance because of the general impact of these emotions on neural circuits and neurotransmitter systems that subserve reward and punishment (see, e.g., Rolls, 1999). This type of emotional influence on decision making - that is, without a specific association between affect and choice-would not be direct evidence to support the somatic marker hypothesis.

To assess the impact of emotion with no predictive relation to decision outcomes, we again used sets of positive, negative, and neutral words to produce emotional reactions that would be independent of GT choice outcomes, but in this case the words did not serve as direct signals of GT choices. Instead, the words were simply maintained during the GT choice trial. Previous work has shown that a digit load of five items maintained in WM can disrupt GT performance and the development of somatic markers (Hinson et al., 2002). We have also demonstrated that verbal buffering of a simple stimulus has no impact on GT performance (Jameson et al., 2004). These findings suggested that holding a single word in a phonological buffer during the GT trial would not interfere with the WM requirements of the task. We only expected an impact on decision making if there was an emotional biasing effect from the words being retained.

\section{Method}

\section{Participants}

The participants were 70 undergraduate students at Washington State University who participated for course credit. They were from 18 to 25 years old and were approximately $60 \%$ female.

\section{Procedure}

The participants were given a brief oral description of the task, paraphrased as follows:

In this experiment, you will be asked to make hypothetical gambles similar to those made in a card game. You will start with a fixed sum of money, and the computer will prompt you to make repeated choices. For each choice, you will sometimes lose money and sometimes win money. Your task is to discover the best choices to make so that at the end of the session you will have the highest amount of money possible. Each choice will be identified with a specific number. When you make your choice on each trial, please press on the appropriate keypad number.

Also, prior to each choice trial, a word will appear briefly on the screen. Please silently rehearse this word so that you will remember it. After each choice, you will be asked to say the word out loud to indicate that you have remembered it.

The participants were seated in front of individual computer terminals and told to begin when ready. A program written in Microsoft Basic provided written instructions and practice trials to illustrate the procedure and collected all the data from the experiment. The participants were given four practice trials to familiarize themselves with the procedure.

Each participant was given 100 GT trials. On each trial, the computer gave a choice among three options. These options were displayed as rectangles, each roughly the area of a playing card, colored red, green, or blue, with the number 1,2 , or 3 in the middle. Outcomes scheduled for good, neutral, and bad options were the same as those for Experiment 1.

Prior to each choice, a word appeared in the center of the computer screen for $2 \mathrm{sec}$ along with the instruction to remember it. The three sets of words - positive, negative, and neutral - used in Experiment 1 were also employed in Experiment 3. After each GT choice, the participant was prompted to say the word aloud.

SCR amplitudes were recorded following the same procedures used in Experiment 1. The timing sequence of each trial was as follows: 1.5 -sec delay-2-sec word presentation-GT choice- $1.5-\mathrm{sec}$ tally display-word said aloud.

The participants were randomly assigned to one of two conditions, positive load or negative load. For the positive load condition, affectively positive words appeared on approximately half of the trials, and affectively negative and neutral words appeared on the other half. For the negative load condition, affectively negative words appeared on approximately half of the trials, whereas affectively positive or neutral words appeared on the other half. The probability of presentation of the predominant word type for a given load condition was .5 , with the other two types assigned a probability of .25 by a pseudorandom process. Thus, the word to be recalled provided no information relevant to outcomes associated with specific choices on a trial. There was simply a different emotional bias that was prevailing during the two load conditions.

One possible source of impact of the positive and negative load conditions was the influence on general mood. To assess changes in mood resulting from the load conditions, half the participants in each load condition were given the Positive and Negative Affect Schedule (PANAS) (see, e.g., Crawford \& Henry, 2004; Watson, Clark, \& Tellegen, 1988) after the GT. The PANAS is a standardized self-report instrument that has been extensively validated in clinical and nonclinical populations and is frequently used as a manipulation check in mood induction studies. The PANAS has 20 self-report items that provide an independent assessment of dispositional positive and negative affect.

\section{Results and Discussion}

Figure 5 summarizes GT performance. Participants rapidly learned to make good choices in the positive load condition. There was only a slight increase in good choices and slight decrease in bad choices across the 


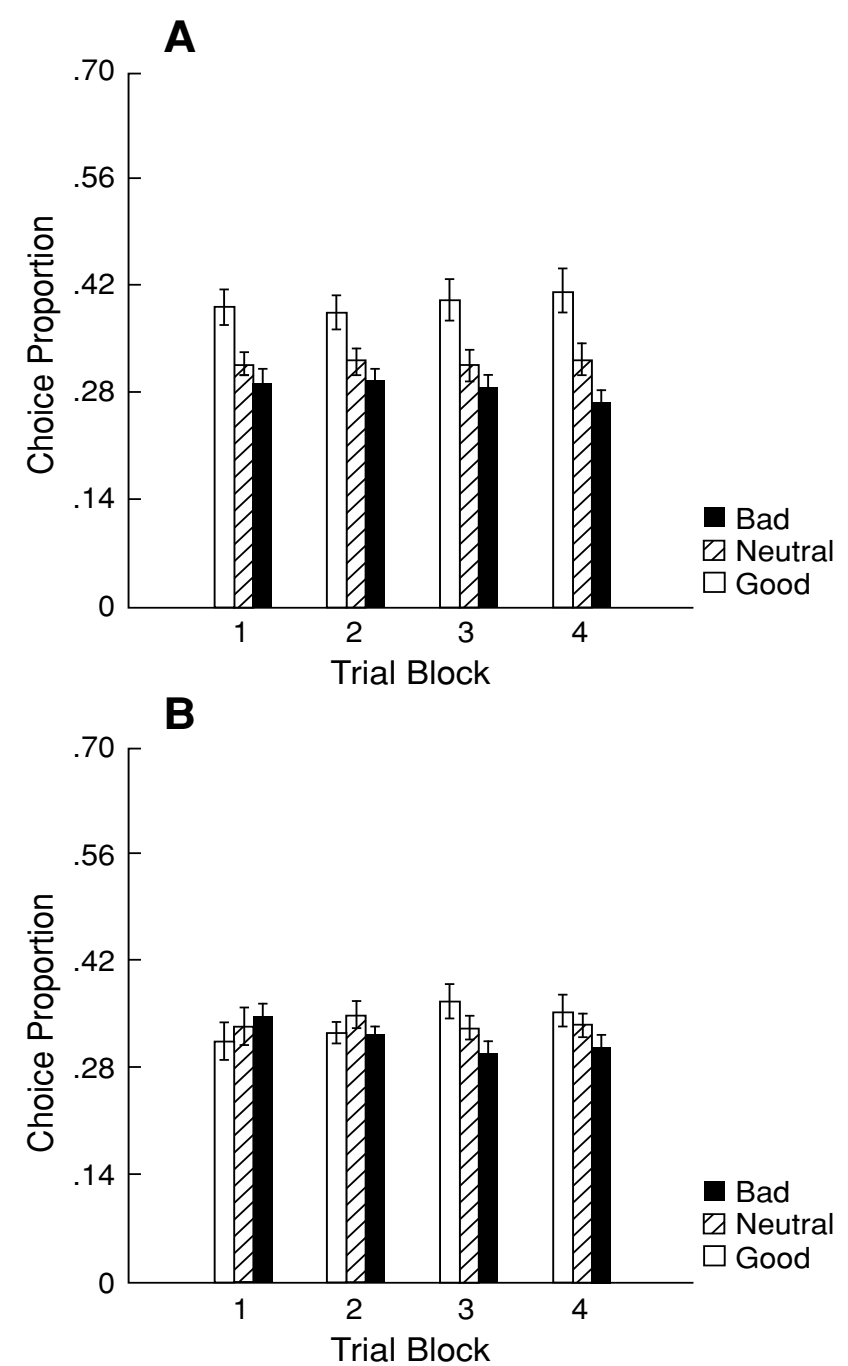

Figure 5. Mean proportion and $S E M$ for the three GT choice types across four blocks of 25 trials in Experiment 3. (A) Positive load condition. (B) Negative load condition.

four blocks of 25 trials. The participants in the negative load condition had great difficulty in making good GT choices. Initial performance was poor and improved only modestly across the session. Statistical reliability of the GT choice results was confirmed by means of a 2 (load condition $) \times 2$ (good or bad choice type $) \times 4$ (trial block) repeated measures ANOVA. The ANOVA indicated a main effect of choice type $[F(1,68)=12.442]$, as well as significant interactions between choice type and load condition $[F(1,68)=5.901]$ and between trial block and choice type $[F(3,204)=2.878]$. Considered alone, good choices reliably increased in the negative load condition $[F(3,102)=2.778]$. But paired $t$ tests showed that good choices never reliably exceeded bad choices in any trial block in the negative load condition. Good choices did not reliably increase across trial blocks in the positive load condition $(F<1)$. Paired $t$ tests indicated that good choices exceeded bad choices in every trial block in the positive load condition. Thus, in the positive load condition, participants were at asymptotic performance by the first block of trials.

Figure 6 shows SCR amplitude prior to the selection of each GT choice. For the negative load condition, overall SCR amplitude is high but there is no differential response to good and bad choices. On the other hand, in the positive load condition differential anticipatory SCR amplitude develops across blocks of trials with SCR to good choices becoming larger than SCR to bad choices. Reliability of SCR results was confirmed by means of a 2 (load condition) $\times 3$ (good, neutral, or bad choice type) $\times 4$ (trial block) repeated measures ANOVA. The ANOVA indicated a main effect of trial block $[F(3,204)=3.375]$, as well as a significant interaction between choice type and load condition $[F(2,136)=5.062]$. Paired $t$ tests showed that SCR amplitude for good and bad choices never differed in the negative load condition. During the positive load con-

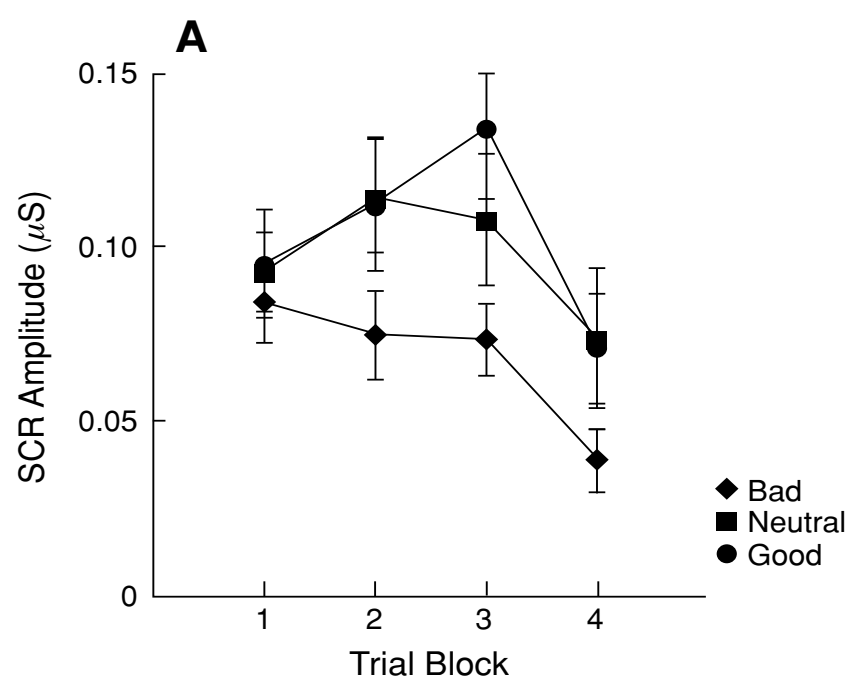

B

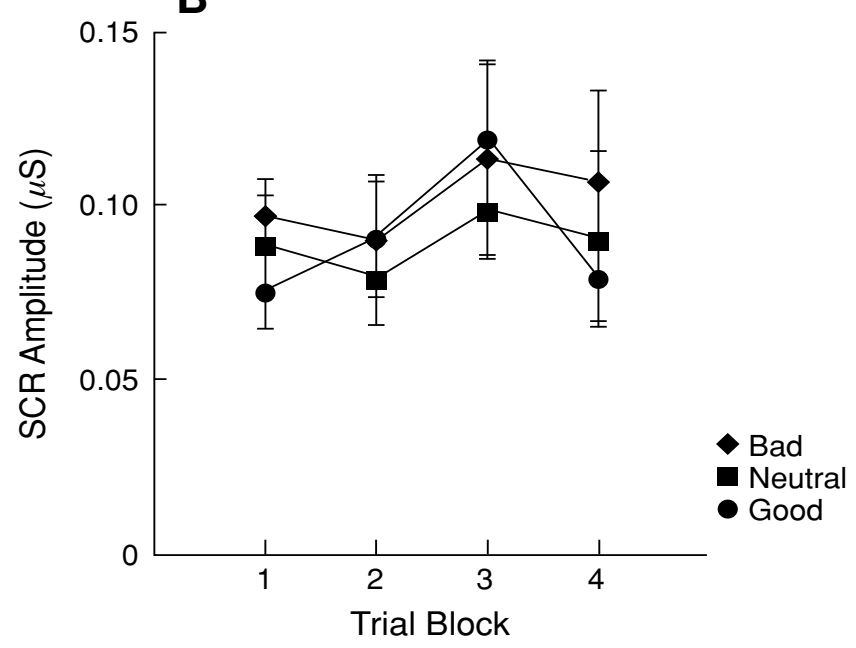

Figure 6. Mean anticipatory SCR amplitude and SEM for the three GT choice types across four blocks of 25 trials in Experiment 3. (A) Positive load condition. (B) Negative load condition. 
dition, there were reliable differences in SCR amplitude for good and bad choices during trial Blocks 3 and 4.

Despite the striking difference in the quality of GT performance between affective loading conditions, there was no evidence of a consistent difference in mood. PANAS scores were analyzed with a 2 (loading condition) $\times 2$ (positive or negative affect scale) repeated measures ANOVA, which revealed no consistent difference in positive or negative affect between loading conditions $(F<1)$. Both positive and negative affect scores were slightly lower than those reported in large-sample validation studies (Crawford \& Henry, 2004).

Despite some differences in detail, the general results for Experiment 3 were similar to those for Experiment 1. Good GT performance was associated with differential affective response to choices. Yet, good GT performance appeared before the development of differential SCR. The positive load condition was similar to the congruent condition - both produced good performance that reached asymptote by the first block of trials. However, asymptotic performance was highest in the congruent condition, with performance in the fourth block of trials being roughly equivalent in the positive load and control conditions. Negative load interfered with the development of good GT performance, though not as much as did incongruent signaling of words and GT choices. Therefore, a direct contingency between affectively valued words and specific choices is not necessary to produce the biasing effect of emotion on GT decision making.

\section{GENERAL DISCUSSION}

The present findings confirm one primary assertion of the somatic marker hypothesis - that emotional reactions can bias decision making. But it was the preexperimental emotional valence of the words that labeled the decks, or that were briefly held in WM, not the emotional reactions to the outcomes of choices from the decks, that strongly influenced GT performance. Choice performance was facilitated when preexisting affective reaction was congruent with choice outcomes, whereas performance was interfered with when preexisting affective reactions were incongruent. A positive affective load enhanced decision making and a negative affective load reduced the quality of decision making.

The somatic marker, as measured by anticipatory SCR to choices, was associated with good GT performance, but it was a lagging, not a leading, indicator. The results presented in Table 2 summarize this general finding. We performed a regression analysis for each block of trials in each experiment. In the analysis, we tried to predict good choices in the block based on SCR amplitude for good, neutral, and bad choices. Table 2 shows at which block in the session, if any-across all studies - SCR was a significant predictor of good choices. SCR always appeared as a predictor at some point in the session for those conditions in which asymptotic GT performance was good. But SCR was never a predictor of good choices in the first block of trials, despite the appearance of good asymptotic GT performance in the conditions of Experiments 1 and 2.

The relationship between anticipatory SCR as an index of the somatic markers and the appearance of discrimination between good and bad GT choices is the key to evaluating the somatic marker hypothesis (Dunn et al., 2006). In early studies, there was a clear connection between SCR and GT performance in VMPFC patients (e.g., Bechara et al., 1997). Anticipatory SCRs appeared in the control group with good GT performance and were absent in the patient group with poor GT performance. Moreover, in the control group, anticipatory SCRs appeared in the session before a stable level of good GT performance was reached. This temporal sequence leads one to the reasonable conclusion that the affective process measured by SCR was a causal factor in decision making.

Our results show that, when presented in the context of affectively biased words, SCR differences develop after good GT performance is obtained. There are several ways in which this result can be interpreted. To begin with, it is possible that SCR is a noisier measure than choice, so that differences among SCRs resolve more slowly. This would mean that the somatic marker is only imperfectly represented by SCR. However, this explanation needs a clarification as to why SCR is sometimes such a striking predictor of GT performance and why in other cases it is not. Second, it is also possible that SCR is a perfectly acceptable measure of the somatic marker but that in this case, the somatic marker develops after good GT performance. Despite its late appearance, the somatic marker might still have a biasing function on choices after the marker has appeared.

A third possibility - the one we find most reasonableis that anticipatory SCR is a good indicator of the somatic marker even though in the present experiments the somatic marker was a reflection of good performance rather than a cause of it. Specifically, the somatic marker may represent an affective reaction to the anticipated outcome

Table 2

Results From Regression Analysis Indicating Blocks Within Session for Which SCR Amplitude Is a Significant Predictor of Good Choices, Along With Adjusted $\boldsymbol{R}^{2}$

\begin{tabular}{cccccc}
\hline Block & E1-Congruent & E1-Incongruent & E2 & E3-Positive & E3-Negative \\
\hline 1 & n.s. & n.s. & n.s. & n.s. & n.s. \\
2 & $.20^{*}$ & n.s. & n.s. & n.s. & n.s. \\
3 & $.18^{*}$ & n.s. & $.03^{*}$ & $.18^{*}$ & n.s. \\
4 & $.20^{*}$ & $.15^{*}$ & $.12^{*}$ & $.09^{*}$ & n.s. \\
\hline Note-n.s., nonsignificant. & ${ }^{*} p<.05$. & & &
\end{tabular}


once a decision has been made. The markers may only appear when the person has enough knowledge of the task to predict choice outcome (cf. Maia \& McClelland, 2004, for a similar view, and Bechara et al., 2005, for a contrary view). The affectively valenced words that we used to bias GT choice had a strong impact on performance. If this impact had been mediated by a somatic marker, we would have expected to see an augmented somatic marker prior to good choice performance in the congruent condition of Experiment 1. Instead, SCR differences lagged behind choice performance.

Furthermore, it appears to us that the data from Experiment 3 do not seem to fit with the notion that the somatic marker is a source of bias or influence on choices. In Experiment 3, there was no association or predictive relation between the affective word occurring prior to the choice and particular choice outcomes as there was in Experiment 1 . Therefore, the affective process produced by the word did not differentiate good and bad options. For example, a good feeling before every choice would not direct a person toward good choices or away from bad choices. In all likelihood, this good feeling would interfere with any putative somatic marker that was directly associated with the choices, making it more difficult to improve decision making. In our initial interpretation of the somatic marker hypothesis, the absence of a predictive relation between word and choice outcomes in Experiment 3 meant that both positive and negative load would interfere with GT performance. Instead we found that negative load interferes with GT performance, whereas positive load facilitates it.

It is not unprecedented for an affective manipulation to have an impact on decision making through something other than a somatic marker. For example, induced emotional states have predictable effects on WM functioning (Gray, 2001). General findings are that positive emotion facilitates verbal WM, whereas negative emotion impairs verbal WM. Also, the DLPFC processes that support $\mathrm{WM}$ are most likely to be influenced by the manipulation of emotion when the WM demands of the task are higher (Perlstein, Elbert, \& Stenger, 2002). It is conceivable that in the present experiments - and especially in Experiment 3-affective manipulations were having an impact on GT performance through their influence on the efficiency of WM functioning. That is, emotional biasing may have reduced or augmented the WM demands of the GT. This idea seems plausible in light of recent evidence that there is no simple partitioning of an affective VMPFC pathway and an independent DLPFC pathway for decision making. Several studies on emotion, WM, and reasoning have concluded that the distinction between hot and cold cognitive pathways is an oversimplification (Gray, Braver, \& Raichle, 2002; Greene, Nystrom, Engell, Darley, \& Cohen, 2004). Previous findings which showed that WM load disrupts the formation of a somatic marker, and the present results which demonstrate the disruption of decision making by preexisting affective bias, cannot easily be explained by the interference with a simple VMPFC pathway to which SCR is sensitive (Jameson et al., 2004).
We conclude, therefore, that poor decision making results from interference with the cognitive processes that are needed to anticipate choice outcomes. Interference with accurate anticipation of outcomes of choices can result from both cognitive loads and emotional biases.

\section{REFERENCES}

BECHARA, A. (2004). The role of emotion in decision-making: Evidence from neurological patients with orbitofrontal damage. Brain \& Cognition, 55, 30-40.

Bechara, A., \& Damasio, A. R. (2005). The somatic marker hypothesis: A neural theory of economic decisions. Games \& Economic Behavior, 52, 336-372.

Bechara, A., Damasio, A. R., Damasio, H., \& Anderson, S. W. (1994). Insensitivity to future consequences following damage to human prefrontal cortex. Cognition, 50, 7-15.

Bechara, A., Damasio, H., \& Damasio, A. R. (2000). Emotion, decision making and the orbitofrontal cortex. Cerebral Cortex, 10, 295307.

Bechara, A., Damasio, H., Tranel, D., \& Damasio, A. R. (1997). Deciding advantageously before knowing the advantageous strategy. Science, 275, 1293-1295.

Bechara, A., Damasio, H., Tranel, D., \& Damasio, A. R. (1998). Dissociation of working memory from decision making within the human prefrontal cortex. Journal of Neuroscience, 18, 428-437.

Bechara, A., Damasio, H., Tranel, D., \& Damasio, A. R. (2005). The Iowa gambling task and the somatic marker hypothesis: Some questions and answers. Trends in Cognitive Sciences, 9, 159-162.

Bechara, A., Dolan, S., \& Hindes, A. (2002). Decision-making and addiction (part II): Myopia for the future or hypersensitivity to reward? Neuropsychologia, 40, 1690-1705.

Bechara, A., Tranel, D., Damasio, H., \& Damasio, A. R. (1996). Failure to respond autonomically to anticipated future outcomes following damage to the prefrontal cortex. Cerebral Cortex, 6, 215-225.

Bradley, M. M., \& LANG, P. J. (1999). Affective norms for English words (ANEW): Stimuli, instructional manual and affective ratings (Tech. Rep. C-1). Gainesville: University of Florida, Center for Research in Psychophysiology.

Crawford, J. R., \& Henry, J. D. (2004). The Positive and Negative Affect Schedule (PANAS): Construct validity, measurement properties and normative data in a large non-clinical sample. British Journal of Clinical Psychology, 43, 245-265.

Damasio, A. R. (1994). Descartes' error: Emotion, reason, and the human brain. New York: Grosset/Putnam.

Damasio, A. R., Tranel, D., \& Damasio, H. (1991). Somatic markers and the guidance of behavior: Theory and preliminary testing. In H. S. Levin, H. M. Eisenberg, \& A. L. Benton (Eds.), Frontal lobe function and dysfunction (pp. 217-229). New York: Oxford University Press.

Dawson, M. E., Schell, A. M., \& Filion, D. L. (2000). The electrodermal system. In J. T. Cacioppo, L. G. Tassinary, \& G. G. Berntson (Eds.), Handbook of psychophysiology (2nd ed., pp. 200-223). Cambridge: Cambridge University Press.

Dolan, R. J. (2002). Emotion, cognition, and behavior. Science, 298, 1191-1194.

Dunn, B. D., Dalgleish, T., \& Lawrence, A. D. (2006). The somatic marker hypothesis: A critical evaluation. Neuroscience \& Biobehavioral Reviews, 30, 239-271.

Fiedler, K., \& Freytag, P. (2004). Pseudocontingencies. Journal of Personality \& Social Psychology, 87, 453-467.

Fiedler, K., Walther, E., Freytag, P., \& Plessner, H. (2002). Judgment biases in a simulated classroom-A cognitive-environmental approach. Organizational Behavior \& Human Decision Processes, $\mathbf{8 8}, 527-561$.

GrAY, J. R. (2001). Emotional modulation of cognitive control: Approach-withdrawal states double-dissociate spatial from verbal two-back task performance. Journal of Experimental Psychology: General, 130, 436-452.

Gray, J. R., Braver, T. S., \& Raichle, M. E. (2002). Integration of emotion and cognition in the lateral prefrontal cortex. Proceedings of the National Academy of Sciences, 99, 4115-4120. 
Greene, J. D., Nystrom, L. E., Engell, A. D., Darley, J. M., \& Cohen, J. D. (2004). The neural bases of cognitive conflict and control in moral judgment. Neuron, 44, 389-400.

Hinson, J. M., Jameson, T. L., \& Whitney, P. (2002). Somatic markers, working memory, and decision making. Cognitive, Affective, \& Behavioral Neuroscience, 2, 341-353.

Jameson, T. L., Hinson, J. M., \& Whitney, P. (2004). Components of working memory and somatic markers in decision making. Psychonomic Bulletin \& Review, 11, 515-520.

Loewenstein, G., \& Lerner, J. S. (2003). The role of affect in decision making. In R. J. Davidson, K. R. Scherer, \& H. H. Goldsmith (Eds.), Handbook of affective sciences (pp. 619-642). Oxford: Oxford University Press.

Maia, T. V., \& McClelland, J. L. (2004). A reexamination of the evidence for the somatic marker hypothesis: What participants really know in the Iowa gambling task. Proceedings of the National Academy of Sciences, 101, 16075-16080.

Manes, F., Sahakian, B., Clark, L., Rogers, R., Antoun, N., AitKEN, M., \& RoBbins, T. (2002). Decision-making processes following damage to the prefrontal cortex. Brain, 125, 624-639.

Perlstein, W. M., Elbert, T., \& Stenger, V. A. (2002). Dissociation in human prefrontal cortex of affective influences on working memoryrelated activity. Proceedings of the National Academy of Sciences, 99, 1736-1741.

Rolls, E. T. (1999). The brain and emotion. Oxford: Oxford University Press.

Rolls, E. T. (2004). The functions of the orbitofrontal cortex. Brain \& Cognition, 55, 11-29.

Tomb, I., Hauser, M., Deldin, P., \& Caramazza, A. (2002). Do somatic markers mediate decisions on the gambling task? Nature Neuroscience, 5, 1103-1104.

Watson, D., Clark, L. A., \& Tellegen, A. (1988). Development and validation of brief measures of positive and negative affect: The PANAS scales. Journal of Personality \& Social Psychology, 54, 1063 1070 .

\section{NOTES}

1. The skin conductance response (SCR) method that we developed in prior studies (Hinson et al., 2002; Jameson et al., 2004) and that we applied in the present study was used with relatively short intertrial intervals. The SCR amplitude measure was based on a peak skin conductance level (SCL) that typically occurred about 4 to $6 \mathrm{sec}$ within the trial. After a choice was made, there was a recovery period that lasted for about $3 \mathrm{sec}$. Typically, the time between choices was about 7 to $9 \mathrm{sec}$. Ideally, a long interval should be used to ensure complete recovery of SCL to baseline. For several reasons, we did not lengthen the time between trials to allow for greater baseline recovery. First, longer intervals between trials would make the 100-choice task tedious, which would lead to fatigue and boredom for the participant and would negatively affect GT performance. Second, our prior results showed that SCRs were fairly well differentiated with the trial intervals we selected. Third, because we were using differential SCR amplitude, some overlap of SCRs across trials would only have been a problem for our measure of SCR amplitude if there had been inconsistent recovery of SCL baseline from one trial to the next.

The only way that incomplete baseline recovery could have produced artifacts in our differential SCR amplitude measure was if there had been some measurable difference in SCL baseline on one trial based on properties of choice on the prior trial. For example, did SCL baseline on one trial differ where the prior trial produced a loss or a gain? Or did SCL baseline vary on one trial depending on whether the choice on the prior trial was good, neutral, or bad? To check these possibilities, we analyzed baseline SCL on a single trial as a function of outcome on the prior trial. Also, we analyzed baseline SCL on a single trial on the basis of whether the prior trial choice was good, neutral, or bad. There was no reliable difference in baseline SCL for any of these cases. Therefore, any fluctuations in baseline SCL were not systematic and could not explain any of the differential SCR amplitude results we have reported.

Finally, even if we had assumed that the time course of the SCR to a trial outcome extended past the baseline into the period when the anticipatory SCR was measured, the carryover of SCR from the previous trial could only have explained one set of results if choices on that trial were made preferentially after good or bad outcomes on the previous trial. We checked to see if there was any predictable relation between outcome on one trial and choice on the following trial by computing the conditional probability of a choice on the following trial as a function of the outcome on the prior trial. The mean probability of choosing a good option was the same, regardless of whether the prior trial resulted in a gain or a loss. The same held true for neutral and bad choices. Therefore, differential carryover of SCR from prior trial outcomes could not be causing the differential anticipatory SCR results we have reported. Because there was no consistent relation between outcome on one trial and the choice on the next, any carryover of SCR from prior trials would act to diminish differential SCR on the following trial.

2. The disruption of GT performance in the incongruent condition was not due to participants choosing only affectively positive words and thereby not sampling the good choices. In the incongruent condition, a total of 9 participants made less than $10 \%$ good choices in the first block of trials. Of these, 6 participants made less than $10 \%$ good choices in Block 2, and 3 participants in this group made less than $10 \%$ good choices in Block 3 . Thus, almost everyone was substantially sampling the good option after two blocks of trials. Even if we exclude this group of 9 participants from the overall analysis, GT performance was still quite poor, with bad choices being the majority in the final block of trials. Therefore, results from the incongruent condition were not due to a small group of people never or very seldom sampling the good option.

3. When one is reporting on a task that has a learning component, overall performance must be broken down into smaller temporal blocks. The convention in GT studies has been to subdivide the session into fourths or fifths, because this temporal grain is fine enough to reveal the changes in choice that occur over time, but it is not so fine grained that there is excessive variability. We did analyze the data with smaller trial blocks, which made the data in the block more variable. Nevertheless, for the congruent condition there was good discrimination between good and bad choices in the first block, even if there were as few as 10 trials per block. And there was never good SCR differential until several blocks into the session. Therefore, the temporal sequence of SCR differentiation following choice differentiation that we report is not dependent on selecting a given number of trials per block for the analysis.

4. To interpret the present data in these terms requires the assumption that the "as if body loop" is operating when people first begin to master the task and that the body loop comes online during later trials. However, it is not clear why the basis of the somatic marker would change during task performance. If there is a change, it seems more likely that the body loop should appear prior to the "as if body loop." (See Bechara \& A. R. Damasio, 2005, pp. 343-344.)

(Manuscript received June 20, 2005; revision accepted for publication March 30, 2006.) 